\title{
Recombinant AAV8-mediated intrastriatal gene delivery of CDNF protects rats against methamphetamine neurotoxicity
}

\author{
Lizheng Wang ${ }^{1}$, Zixuan Wang ${ }^{1}$, Xiaoyu $\mathrm{Xu}^{2}$, Rui Zhu ${ }^{1}$, Jinpeng Bi ${ }^{1}$, Wenmo Liu ${ }^{1}$, Xinyao Feng ${ }^{1}$, Hui $\mathrm{Wu}^{1}$, \\ Haihong Zhang1, Jiaxin $W^{1}{ }^{1}$, Wei Kong1,3, Bin Yu ${ }^{1 凶}$, Xianghui Yu1,3凶 \\ 1. National Engineering Laboratory for AIDS Vaccine, School of Life Sciences, Jilin University, Changchun 130012, China; \\ 2. Evidence Identification Center, Department of Public Security of Jilin Province, Changchun 130051, China; \\ 3. Key Laboratory for Molecular Enzymology and Engineering, the Ministry of Education, School of Life Sciences, Jilin University, Changchun 130012, China. \\ $\square$ Corresponding authors: Address: School of Life Sciences, Jilin University, Changchun 130012, China. Fax: +86 043185167751 E-mail addresses: \\ yubin@jlu.edu.cn (B. Yu), xianghui@jlu.edu.cn (X. Yu)
}

(1) Ivyspring International Publisher. This is an open access article distributed under the terms of the Creative Commons Attribution (CC BY-NC) license (https://creativecommons.org/licenses/by-nc/4.0/). See http://ivyspring.com/terms for full terms and conditions.

Received: 2016.12.05; Accepted: 2017.01.31; Published: 2017.03.17

\begin{abstract}
Methamphetamine (METH) exerts significant neurotoxicity in experimental animals and humans when taken at high doses or abused chronically. Long-term abusers have decreased dopamine levels, and they are more likely to develop Parkinson's disease (PD). To date, few medications are available to treat the METH-induced damage of neurons. Glial cell line-derived neurotrophic factor (GDNF) has been previously shown to reduce the dopamine-depleting effects of neurotoxic doses of METH. However, the effect of cerebral dopamine neurotrophic factor (CDNF), which has been reported to be more specific and efficient than GDNF in protecting dopaminergic neurons against 6-OHDA toxicity, in attenuating METH neurotoxicity has not been determined. Thus, the present study aimed to evaluate the neuroprotective effect of CDNF against METH-induced damage to the dopaminergic system in vitro and in vivo. In vitro, CDNF protein increased the survival rate and reduced the tyrosine hydroxylase $(T H)$ loss of METH-treated PCl2 cells. In vivo, METH was administered to rats following human CDNF overexpression mediated by the recombinant adeno-associated virus. Results demonstrated that CDNF overexpression in the brain could attenuate the METH-induced dopamine and TH loss in the striatum but could not lower METH-induced hyperthermia.
\end{abstract}

Key words: Methamphetamine, Neurotoxicity, Adeno-associated virus, CDNF, Neuroprotection, Dopamine.

\section{Introduction}

The United Nations Office of Drug and Crime estimated that between 13.94 and 53.81 million people used an amphetamine-like substance at least once in 2012, and the number of abusers has been increasing in the past 10 years (World Drug Report 2014). Methamphetamine (METH), a derivative of amphetamine, is abused worldwide [1]. When taken at high doses or chronically, METH may result in significant damage in the brain, especially to the dopaminergic system. Long-term abusers have decreased levels of dopamine and dopamine transporters as well as prominent microglial activation in the striatum and other areas of the brain, changes similar to those observed in Parkinson's disease (PD) patients [2]. Furthermore, METH users are almost two to three folds as likely as non-users to develop PD, even though METH abuse and PD have distinct symptomatic profiles [2, 3]. However, few specific medications counteracting the neurotoxicity of METH are available.

Cerebral dopamine neurotrophic factor (CDNF) is a member of the MANF family of proteins and is abundantly produced in various brain tissues of adult mice, such as the cortex, hippocampus, substantia nigra (SN), cerebellum and locus coeruleus [4]. It appears to be more specific and effective for dopaminergic neurons than other known trophic factors, such as glial-derived neurotrophic factor 
(GDNF) $[5,6]$ and brain-derived neurotrophic factor (BDNF) [7]. Human CDNF protein injected into the rat striatum has been shown to exhibit a substantial neuroprotective effect against 6-OHDA lesions and improve the abnormal behavior of animals [4, 8]. Furthermore, adeno-associated virus 2 (AAV2)mediated gene delivery of human CDNF into the rat brain has been demonstrated to exert a long-term protective effect on dopaminergic neurons in 6-OHDA-induced PD rat models [9]. Moreover, CDNF was suggested to have great therapeutic potential in treating human PD patients.

GDNF and other neurotrophic factors belonging to the GDNF family are reported as potential effective candidates for attenuating METH neurotoxicity in the dopaminergic system. They have been found effective in increasing the striatal dopamine content of METH-treated rats [10, 11] and persistently attenuating METH self-administration and relapse in mice [13]. However, the neuroprotective effect of CDNF against METH toxicity has been unknown. Thus, this study mainly aimed to determine the effect of CDNF on METH-induced neurotoxicity in vitro and in vivo.

\section{Materials and methods}

\section{Animals}

Adult male Wistar rats weighing 180-250 g were used for all experiments. They were housed three per cage under a $12 \mathrm{~h}$ light/dark cycle in a temperature-controlled room $\left(21-23^{\circ} \mathrm{C}\right)$. Food and water were available ad libitum. All animal procedures were conducted in strict accordance with the National Institutes of Health Guide for the Care and Use of Laboratory Animals and were approved by the University Committee on the Use and Care of Animals of Jilin University of China. All surgeries were performed under anesthesia, and all efforts were made to minimize suffering of animals.

\section{Cell lines}

PC12 cells were used as a model of dopaminergic neurons in vitro, and HEK 293T cells were used to produce CDNF protein and recombinant adeno-associated virus (rAAV) vectors. Both cell lines were purchased from ATCC and were routinely maintained in Dulbecco's modified Eagle medium containing $10 \%(\mathrm{v} / \mathrm{v})$ fetal bovine serum (FBS), 100 $\mathrm{U} / \mathrm{mL}$ penicillin and $100 \mu \mathrm{g} / \mathrm{mL}$ streptomycin at $37^{\circ} \mathrm{C}$ in a humidified atmosphere of $95 \%$ air and $5 \% \mathrm{CO}_{2}$. The culture medium was changed every two or three days.

\section{Production of rAAV vectors}

The cDNA encoding human CDNF or red fluorescent protein (RFP) was cloned into the pAAV-CAG-MCS vector. The rAAV packaging method has been reported previously [12]. The titers of the rAAV vectors, named AAV8-CDNF and AAV8-RFP, were $2.8 \times 10^{13}$ and $1.8 \times 10^{13} \mathrm{vg} / \mathrm{mL}$ respectively.

\section{Preparation of CDNF proteins}

The human CDNF gene was constructed and cloned into the VR1012 vector. The resulting plasmid designated as pVR1012-CDNF-his-tag was transfected into HEK 293T cells to express and secrete CDNF proteins into the medium with the help of a signal peptide. The medium was harvested $72 \mathrm{~h}$ post transfection for further purification of CDNF proteins using Ni-NTA spin columns. The purity of the CDNF proteins surpassed $90 \%$, reaching $0.4 \mathrm{mg} / \mathrm{mL}$ after concentration.

\section{PC1 2 cell survival and TH levels}

PC12 cells were incubated with CDNF $(1 \mu \mathrm{M})$ for $4 \mathrm{~h}$ and then with METH (4 mM) dissolved in medium without FBS. PC12 cell survival was measured by the MTT assay at $24 \mathrm{~h}$ post METH incubation. For TH expression detection, PC12 cells were pre-incubated with CDNF $(1 \mu \mathrm{M})$ followed by the addition of METH $(2 \mathrm{mM})$. At $24 \mathrm{~h}$ post METH incubation, PC12 cells were harvested to detect TH levels by Western blot.

\section{Stereotaxic microinjections}

Rats were anesthetized by intraperitoneally (i.p.) administered pentobarbital sodium $(60 \mathrm{mg} / \mathrm{kg})$. The rAAV8 vector (AAV8-CDNF) or PBS was unilaterally injected into the right striatum using two coordinates relative to the bregma and dura: $(\mathrm{A} / \mathrm{P}+0.5 \mathrm{~mm}, \mathrm{~L} / \mathrm{M}$ $-2.0 \mathrm{~mm}, \mathrm{D} / \mathrm{V}-5.0 \mathrm{~mm})$ and $(\mathrm{A} / \mathrm{P}+0.5 \mathrm{~mm}, \mathrm{~L} / \mathrm{M}-3.8$ $\mathrm{mm}, \mathrm{D} / \mathrm{V}-5.0 \mathrm{~mm}$ ) according to the Paxinos and Watson's rat brain atlas (Figure 2A). The viral vector $\left(1 \times 10^{10} \mathrm{vg}\right.$ in $2 \mu \mathrm{L}$ PBS$)$ or PBS alone $(2 \mu \mathrm{L})$ was microinjected into each site at a rate of $0.25 \mu \mathrm{L} / \mathrm{min}$ using a syringe pump. The syringe was left in place for $8 \mathrm{~min}$ and then raised slowly out of the brain over $2 \mathrm{~min}$.

\section{METH administration}

In this study, rats repeatedly given high-dose METH were used as models. Three weeks after microinjection of rAAV8 vector or PBS, rats were randomly divided into four groups designated as follows: AAV8-CDNF/METH (C/M), vehicle/METH $(\mathrm{V} / \mathrm{M}), \mathrm{AAV} 8-\mathrm{CDNF} /$ saline $(\mathrm{C} / \mathrm{S})$ and vehicle/saline (V/S). Animals were injected (i.p.) with either METH $(7.5 \mathrm{mg} / \mathrm{kg} \times 4)$ or saline $(1 \mathrm{~mL} / \mathrm{kg} \times 4)$ at $2 \mathrm{~h}$ intervals. Rectal temperature was taken during the process. 


\section{Tissue collection and high performance liquid chromatography (HPLC) analysis}

At the end of the experiments, the animals were killed by decapitation while still anesthetized with pentobarbital sodium. The brains were removed rapidly and chilled in ice-cold saline. The whole brain was separated into two hemispheres to distinguish the injected side and the non-injected side. The striatum was dissected from each half of the slice as a single piece. The tissue pieces were placed in pre-weighed vials, weighed and frozen in liquid nitrogen. Samples were stored at $-80^{\circ} \mathrm{C}$ until assayed by HPLC.

For determining dopamine content, the samples were sonicated in $300 \mu \mathrm{L}$ of cold $0.1 \mathrm{M}$ perchloric acid containing dihydroxybenzylamine as an internal standard. After centrifuging samples for $5 \mathrm{~min}$ at $13,500 \times g$, the supernatant was diluted with HPLC mobile phase and injected $(50 \mathrm{~mL})$ onto the HPLC column. The mobile phase consisted of sodium acetate $(0.1 \mathrm{M})$, EDTA $(0.1 \mathrm{mM})$ and methanol (10\%) and was adjusted to $\mathrm{pH} 5.1$ with glacial acetic acid, filtered and degassed. A C18 column (150 mm × $4.6 \mathrm{~mm}$ i.d., $5 \mu \mathrm{m})$ was used for separations with a flow rate of 0.7 $\mathrm{mL} / \mathrm{min}$.

\section{Immunohistochemistry}

Seven days after METH or saline treatment, rats were anesthetized and perfused with saline and $4 \%$ paraformaldehyde in $0.01 \mathrm{M}$ PBS. Brains were removed, postfixed in $4 \%$ paraformaldehyde overnight and then dehydrated with $20 \%$ gradient and $30 \%$ gradient sucrose-PBS solutions. Brain tissues were frozen in isopentane and kept at $-80^{\circ} \mathrm{C}$ for further immunohistochemical procedures. To avoid confusion of the AAV8-CDNF-injected and non-injected brain hemispheres, the two hemispheres of each brain were separated before embedding and then separately frozen.

Coronal brain sections (30 $\mu$ m-thick) from the entire substantia nigra pars compacta $(\mathrm{SNc})$ and a large part of the striatum were prepared on a slicing vibratome and collected in a 6-well plate. Every 6th section of a brain was collected in a well. Sections containing the $\mathrm{SNc}$ and striatum were selected according to Paxinos \& Watson's rat brain atlas, with the rostro-caudal extent of the $\mathrm{SNc}$ being between $-4.8 \mathrm{~mm}$ and $-6.12 \mathrm{~mm}$ and the rostro-caudal extent of the striatum being between $+2.16 \mathrm{~mm}$ and -0.24 $\mathrm{mm}$ with respect to the bregma. Free-floating staining procedures were conducted to reveal $\mathrm{TH}$ and CDNF using an immunoperoxidase. Briefly, sections were placed in citrate buffer to retrieve antigens and washed in PBS-Triton X-100 (0.2\%) for $3 \times 10 \mathrm{~min}$ and then rinsed in $0.3 \%$ hydrogen peroxide to quench the endogenous peroxide. Appropriate primary antibodies [mouse anti-TH (diluted 1:200, Sigma) or rabbit anti-CDNF (diluted 1:500, Abcam)] were incubated with sections at $4^{\circ} \mathrm{C}$ overnight. The horseradish peroxidase (HRP)-labeled secondary antibodies were developed by a peroxidase reaction with diaminobenzidine (DAB) as the chromagen. Three representative sections per rat were used in quantifications.

\section{Western blotting analysis}

The Western blotting analysis was conducted as in our previous work [13]. TH expression was quantified with respect to the signals of the corresponding GAPDH band.

\section{Statistical analysis}

One-way ANOVA followed by the Newman-Keuls test was performed to analyze significance differences between groups. All data are expressed as the mean \pm SEM, and the statistical significance level was set at $P<0.05$.

\section{Results \\ CDNF protects METH-treated PC1 2 cells in vitro}

$\mathrm{TH}$, as a marker of dopaminergic neurons, is an enzyme playing a decisive role in the synthesis of dopamine. The PC12 cell line containing endogenous $\mathrm{TH}$ expression [14] was chosen to evaluate the neurotoxicity of METH in vitro. Cell survival of different doses of METH-treated PC12 cells was detected by the MTT assay (Figure 1A). Cell survival decreased as the METH dose increased. The remaining cells were less than $40 \%$ at the METH dose of $6 \mathrm{mM}$ and approximately $56 \%$ at the dose of $4 \mathrm{mM}$; however, cell survival improved clearly to $86 \%$ in 4 mM METH-treated PC12 cells when pre-incubated with CDNF (Figure 1B). Meanwhile, the TH level of 2 $\mathrm{mM}$ METH-treated cells pre-incubated with CDNF was increased by $24 \%$ compared with $2 \mathrm{mM}$ METH-treated cells without CDNF (Figure 1C, 1D). These results provided primary evidence of the effect of CDNF against METH neurotoxicity in vitro.

\section{In vivo experimental procedures}

Based on the results in vitro, the neuroprotective effect of CDNF was further evaluated in the rat brain. In brief, rats were microinjected with the AAV8-CDNF vector in the brain for overexpression of the protein, and then high-dose METH was administered. Finally, levels of dopamine and $\mathrm{TH}$ were detected.

For the injection of the rAAV vectors, we used two neighboring injection sites (Figure 2A). We 
previously found that the transgene expression mediated by rAAV spread more efficiently using two

A

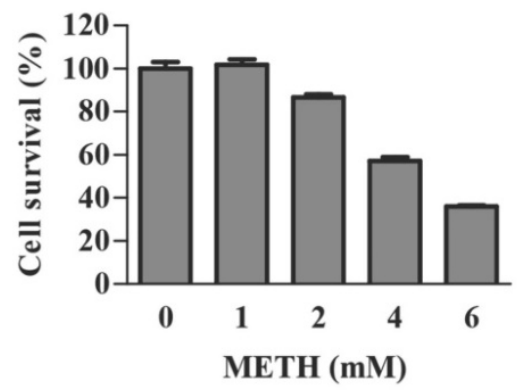

C

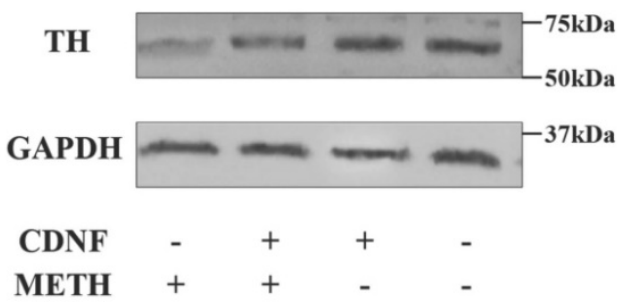

neighboring injection sites than using a single injection site (data not shown).

B

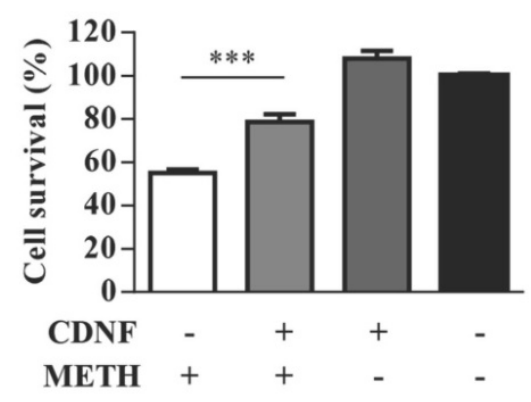

D
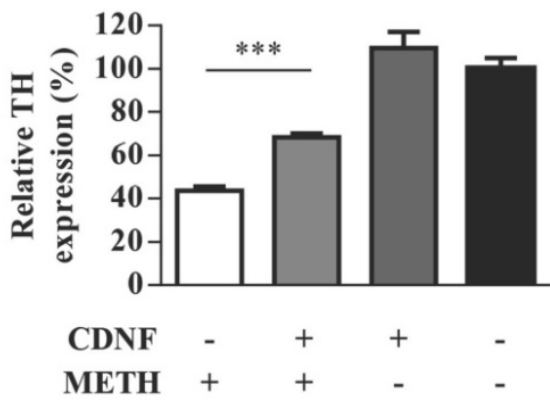

Figure 1. CDNF protects PC12 cells against METH toxicity in vitro. (A) Effect of METH on cell survival. PC12 cells were exposed to varying doses of METH for $24 \mathrm{~h}$. Cell viability compared with untreated cells was evaluated by the MTT assay $(n=5)$. (B) Effect of CDNF on cell survival. METH (4 mM) was used to injure $\mathrm{PC} 12$ cells, which were concomitantly incubated with or without CDNF protein as indicated. Cell survival compared with untreated cells was detected by the MTT assay after $24 \mathrm{~h}(\mathrm{n}=5)$. (C) Effect of CDNF on TH expression in PC12 cells as analyzed by Western blot. Representative results are shown. Cells were processed with the same method as that to evaluate the effect on cell survival except that the dose of METH here was $2 \mathrm{mM}(\mathrm{n}=3$ ). (D) Relative expression was evaluated by quantifying Western blot bands. $* * * p<0.001$.

A

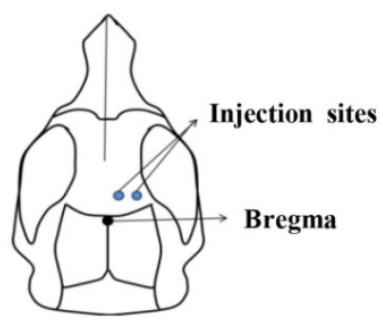

B

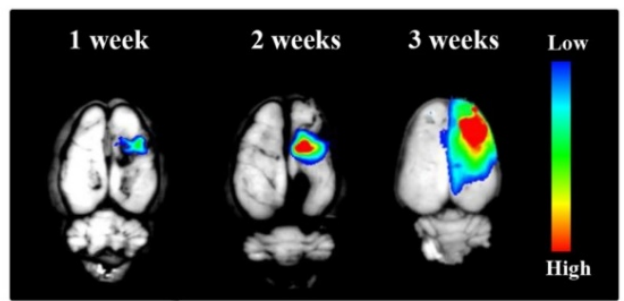

D

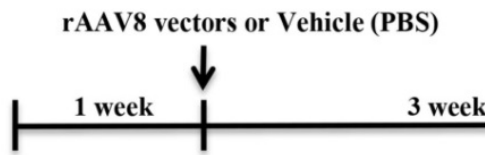

Rats arrival Stereotaxic microinjections

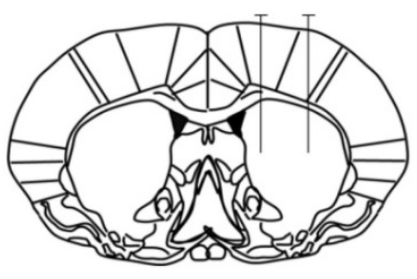

C

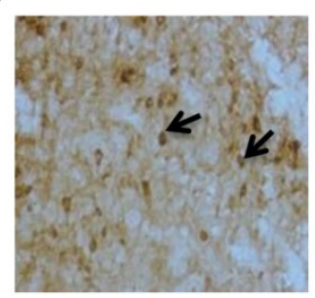

METH or Saline

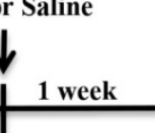

Decapitation or perfusion

Figure 2. rAAV8-mediated transgene expression and animal experimental design. (A) Two sites for microinjection of rAAV8 vectors were used. (B) AAV8-mediated reporter gene (RFP) expression in the rat brain. RFP expression was detected at different time points post microinjection. (C) CDNF expression in the rat striatum was detected 3 weeks post microinjection by immunostaining. (D) Design of animal experiment. Rats were treated with METH three weeks post microinjection with rAAV8 vectors. One week later, all animals were killed for detection of dopamine or TH. 
Before administration of the AAV8-CDNF vector, the AAV8-RFP vector was injected into the rat striatum to determine the variation in transgene expression over time. The rAAV8-mediated RFP (as a reporter) expression was observed using the In-Vivo Imaging System Fx Pro system (Kodak Carestream Health, Rochester, NY, USA). The rAAV8-mediated RFP expression increased with time (Figure 2B). The transgene expressed well, and RFP spread widely around the brain injection sites three weeks post viral injection. As expected, CDNF was well-detected by immunostaining after three weeks (Figure 2C). These observations indicated that AAV8-CDNF mediated CDNF expression in the rat brain would be sufficient for subsequent experiments, and METH was determined to be administered at three weeks post AAV8-CDNF microinjection.

During the repeated METH injections, rectal temperatures were measured. All rats were killed at the 7th day post METH administration for evaluating the neuroprotection of CDNF. The whole animal experimental design is shown in Figure 2D.

\section{Rectal temperature during METH administration}

METH-caused hyperthermia is known to increase the toxicity of the drug [10]. To examine whether CDNF can influence METH-induced hyperthermia, the rectal temperature of CDNF-overexpressing rats was measured during METH administration (Figure 3). METH administration increased the rectal temperature acutely; however, CDNF overexpression seemed unable to attenuate the hyperthermia. Hence, the neuroprotective effect of CDNF against METH was not determiend to involve controlling the high core body temperature.

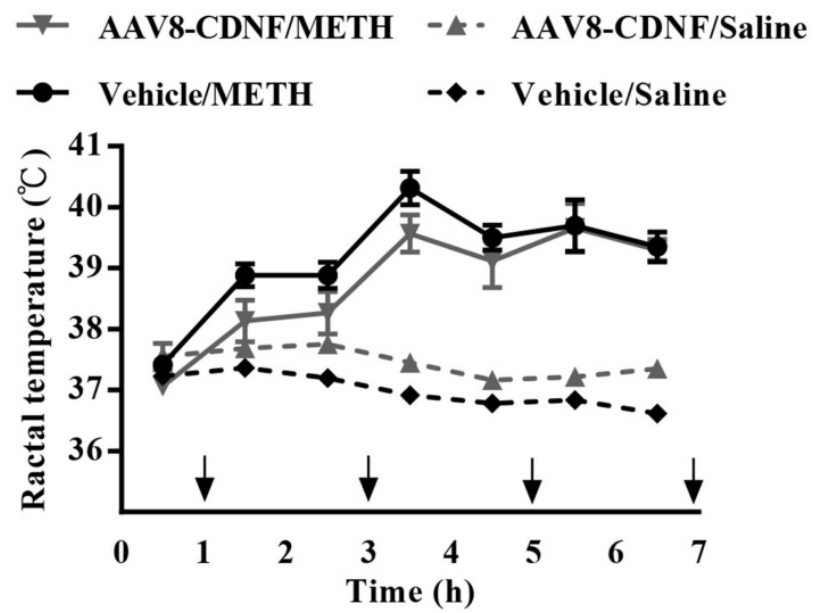

Figure 3. Rectal temperature of METH-injected rats. Animals received four repeated injections of METH. During the intervals, rectal temperature was measured. Temperatures of all METH-treated rats significantly rose over the course of the experiment.

\section{Evaluation of effect of CDNF in METH-induced striatal dopamine loss}

METH administration has been shown to cause increased dopamine secretion within a short amount of time and significantly decrease the dopamine level several days later $[15,16]$. At the end of the animal experiment in this study, 4-5 rats of every group were decapitated, and dopamine levels in the striatum were quantified by HPLC (Figure 4). The vehicle/METH group showed the lowest dopamine level (approximately half of normal) detected in the striatum of both hemispheres. Dopamine levels of both groups administered with saline instead of METH showed no difference between the two hemispheres. In the AAV8-CDNF/METH group, the rat brain hemisphere not overexpressing CDNF showed approximately half the dopamine level of that overexpressing CDNF, similar to that of the vehicle/METH group. Meanwhile, the CDNF-overexpressed striatum contained a significantly increased dopamine level compared with the hemisphere not overexpressing CDNF and both hemispheres of the vehicle/METH group. CDNF overexpression increased the dopamine level to $82.2 \%$, while the striatum without CDNF overexpression maintained an approximately 50\% dopamine level due to the METH neurotoxicity.

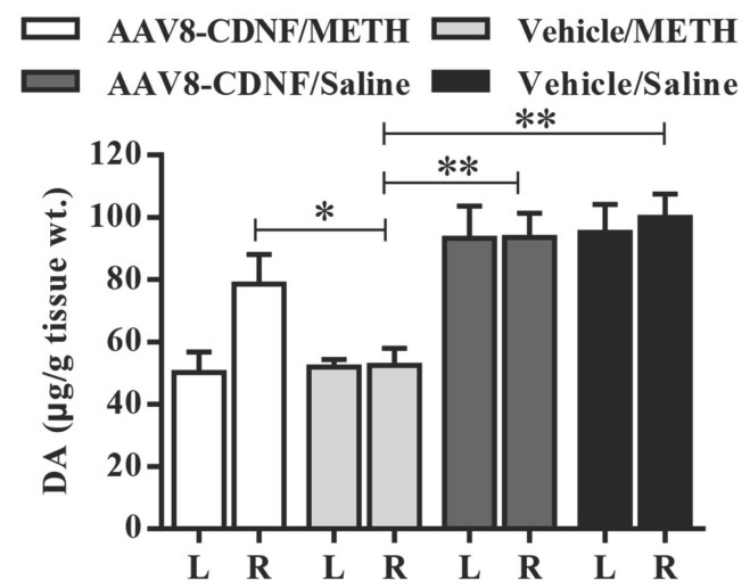

Figure 4. Dopamine content in rat striatum. The striatum of each rat was isolated at the 7th day after METH administration. Dopamine content was quantified by HPLC ( $n=4-5)$. ' $L$ ' indicates left striatum, and ' $R$ ' indicates right striatum. The dopamine content per gram tissue was analyzed in all groups. $* P<$ $0.05, * * P<0.005$

\section{Evaluation of effect of CDNF in METH-induced TH loss}

Striatal TH density was determined using immunohistochemical analysis, and the $\mathrm{TH}$ expression was quantified by using Western blot. As shown in Figure 5A, CDNF overexpression indeed attenuated $\mathrm{TH}$ loss in the striatum. By quantifying the 
density of images, $\mathrm{TH}$ immunoreactivity of the CDNF-overexpressed striatum was determined to be $89.4 \%$, while that in the vehicle/METH group was $53.5 \%$ (striatum TH-immunoreactivity of vehicle/saline group was set at 100\%) (Figure 5B). To further determine the effect of CDNF in attenuating striatal $\mathrm{TH}$ loss, Western blot was used to quantify $\mathrm{TH}$ expression in the striatum (Figure 5C, 5D), and the results were consistent with those of $\mathrm{TH}$ immunostaining.

$\mathrm{TH}$ staining in the SNc region revealed a slight METH-induced decrease of TH-positive cells (Figure
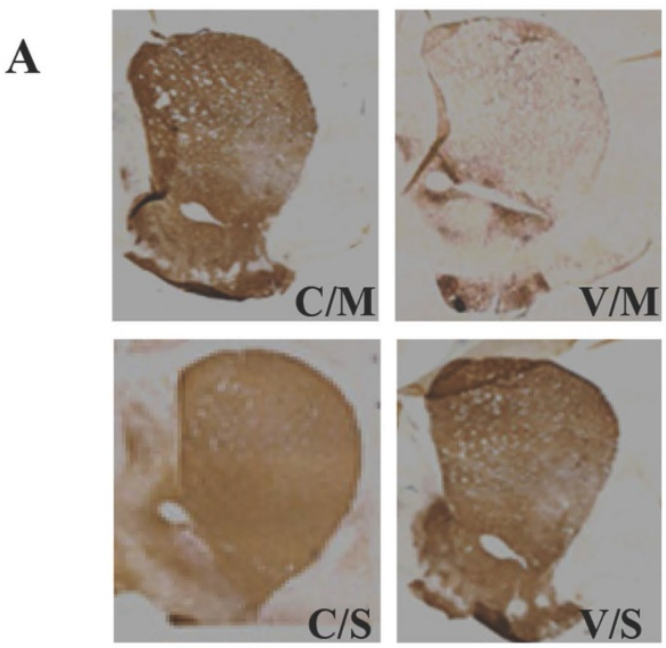

C

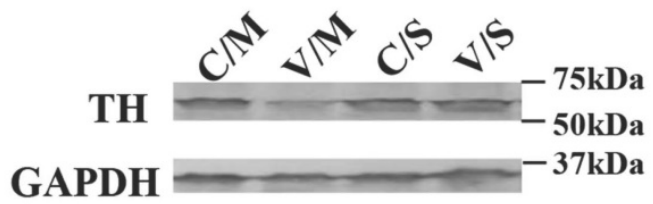

$\mathbf{E}$

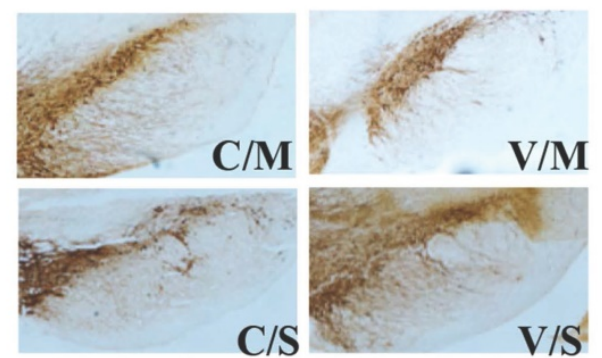

$5 \mathrm{E}, 5 \mathrm{~F})$. The results were similar to those of previous reports, in which METH was shown to influence dopaminergic markers only slightly in the SN [17-21]. Nevertheless, the effect of CDNF in attenuating the slight TH decrease in the SNc could be seen, although the TH-positive numbers of all groups also showed no significant differences. Relative to the vehicle/saline group set at $100 \%$ as a standard, percentages of TH-positive cells were $106.52 \%$ in the AAV8-CDNF/METH group, $91.61 \%$ in the vehicle/METH group and $109.08 \%$ in the AAV8-CDNF/saline group.
B

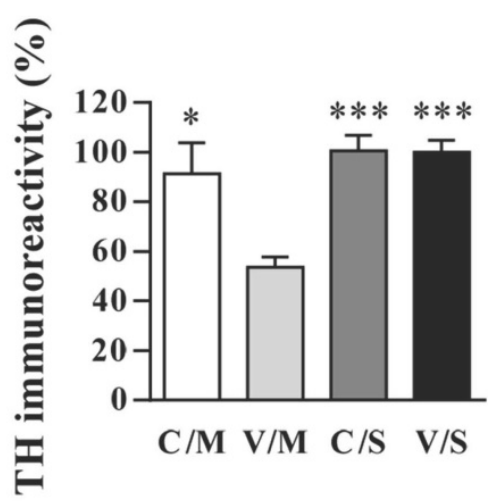

D
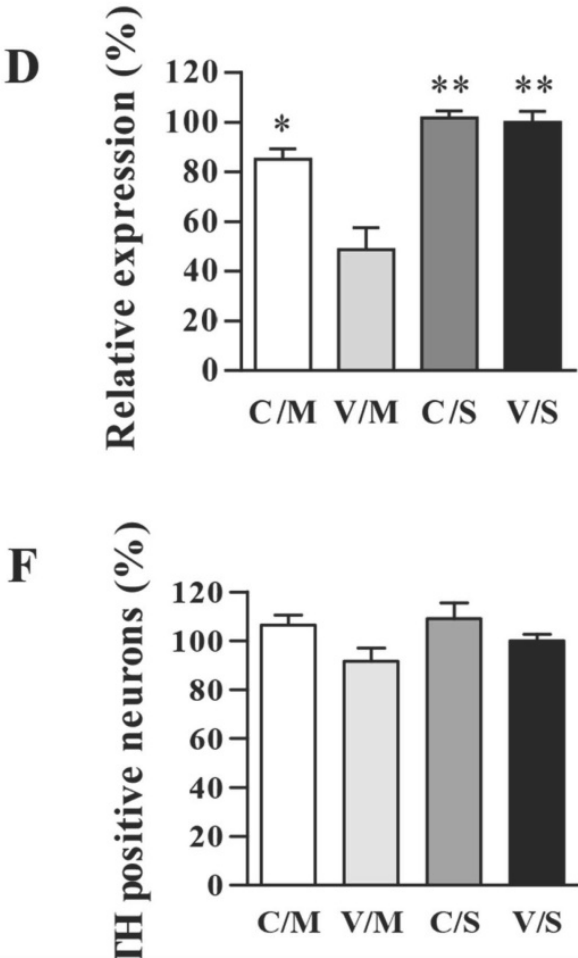

Figure 5. Effects of CDNF on METH-induced decreases in TH expression. (A) Representative images of TH-stained striatum are shown. (B) Analysis of striatal TH expression ( $n=4-6)$ was performed using Image-pro-plus-6.0 software. $P$-values were obtained by comparing with the right striatum of the vehicle/METH group. (C) TH expression in the right striatum (injected side) was analyzed by Western blot. The relative expression was evaluated by quantifying Western blot bands $(n=3)$. (D) Representative images of TH-stained SNc are shown. (E) TH-positive cells (dark brown spots) in the SNc of the injected side of the brain were counted $(n=4-6)$. No significant differences were found in any of the groups when compared with the vehicle/saline group. C/M: AAV8-CDNF/METH group; V/M: vehicle/saline group; C/S: AAV8-CDNF/saline group; V/S: vehicle/saline group. $* P<0.05$, $* * P<0.005, * * * P<0.001$. 


\section{Discussion}

METH is known to cause significant neurotoxicity in the dopaminergic system and increase the risk of developing PD. The insufficiency of drugs to efficiently attenuate the toxicity of METH is a significant problem. GDNF and other neurotrophic factors belonging to the GDNF family have been reported as potentially effective candidates for attenuating METH neurotoxicity in the dopaminergic system [22, 23]. However, the effect of CDNF, which has similar or even better neuroprotective effects against 6-OHDA toxicity, in preventing METH-induced neurotoxicity has not been determined previously. Therefore, we mainly aimed here to determine the effect of CDNF in attenuating the damage to the dopaminergic system induced by METH in vitro and in vivo.

The PC12 cell line was used for investigating the effect of CDNF in attenuating the METH-induced toxicity in vitro. Millimolar concentrations of METH can cause apoptosis of PC12 cells in vitro [24]. In this study, we also found that METH was only able to influence the viability and TH loss of PC12 cells at millimolar but not lower (e.g., micromolar) concentrations. Although the micromolar and millimolar concentrations of METH are higher than that found physically in the brain after systemic administration, the cell model treated with METH would still reflect the relative toxicity effects of the drug. The analysis of cell viability and $\mathrm{TH}$ loss in vitro showed that CDNF was able to attenuate the toxicity caused by METH.

The in vitro experiments offered primary evidence of the CDNF neuroprotective effect in attenuating METH-induced neurotoxicity. Further investigation of the protective effect of CDNF against METH toxicity was performed in vivo. The rAAV8 viral vector was chosen to deliver the CDNF gene into the rat striatum by microinjection, as AAV8 was shown to be a relatively efficient vector for gene transfer in the brain [25]. In contrast to the previous studies $[22,26]$ in which proteins were directly perfused into the rat brain to explore the neuroprotection of GDNF against METH, the single injection of AAV8-CDNF ensured stable expression of CDNF during the METH-induced injury.

At seven days post METH administration, the dopamine level in the rat striatum was detected, and immunochemical analysis of $\mathrm{TH}$ expression in the striatum and $\mathrm{SNc}$ was performed. The results indicated that overexpression of CDNF attenuated the loss of dopamine (Figure 4) and TH (Figure 5) in the rat brain. Although the detailed mechanism of CDNF in attenuating the METH-induced neurotoxicity was not determined here, the underlying process may occur through alleviating endoplasmic reticulum stress-induced cell damage and inflammatory cytokine secretion, similar to the effect of CDNF overexpression in astrocytes [27]. CDNF was also found to significantly attenuate the production of proinflammatory cytokines (PGE2 and IL-1 $\beta$ ) and remarkably alleviate the cytotoxicity in LPS-induced microglia by suppressing the phosphorylation of JNK [28]. When exploring its effect on preventing the apoptosis of PC12 cells induced by 6-OHDA, CDNF was considered to exert its role via modulating Bcl-2/Bax and caspase-3 activation [29]. These studies indicate the possible mechanisms of CDNF-mediated protection of neurons against $\mathrm{METH}$, but the precise means by which it confers these effects may be complicated and will require further exploration.

Collectively, the findings in this study indicate that CDNF has the potential to be the target in therapeutic strategies to mitigate the harmful effects of acute METH overdoses as well as the effects of chronic METH use on the CNS in human abusers. As chronic abuse of METH is likely to cause permanent neurological damage, using $\mathrm{rAAV} 8$ vectors with the capacity for long-term transgene expression to mediate CDNF gene delivery may be a more suitable choice than direct delivery of these corresponding proteins, which would result in only short-term therapeutic effects. In addition, CDNF may protect the dopaminergic system against METH neurotoxicity through a different pathway from that of GDNF, considering the distinct structures of both neurotrophic factors. Future studies may also explore the combination of both neurotrophic factors to treat METH-induced neuro-damage as an alternative approach for enhancing therapeutic effects.

\section{Abbreviations}

METH, methamphetamine; CDNF, cerebral neurotrophic factor; $\mathrm{SNc}$, substantia nigra compacta; CNS, central nervous system; PD, Parkinson's disease

\section{Acknowledgments}

This study was supported by the National Natural Science Foundation of China (No. 81472816, 31300756), the Key Projects in the National Science \& Technology Pillar Program in the Twelfth Five-year Plan Period (Grant 2012ZX10001-009), the Science \& Technology Development Plan of Jilin Province (No. 20160101240JC) and Graduate Innovation Fund of Jilin University (No. 2016151). We also wish to acknowledge Phuong Thi Sarkis for editorial support in the preparation of this manuscript. 


\section{Competing Interests}

The authors have declared that no competing interest exists.

\section{References}

1. Rawson RA, Gonzales R, McCann M, Ling W. Use of methamphetamine by young people: is there reason for concern? Addiction. 2007; 102: 1021-2.

2. Granado N, Ares-Santos S, Moratalla R. Methamphetamine and Parkinson's disease. Parkinson's disease. 2013; 2013: 308052.

3. Curtin K, Fleckenstein AE, Robison RJ, Crookston MJ, Smith KR, Hanson GR. Methamphetamine/amphetamine abuse and risk of Parkinson's disease in Utah: a population-based assessment. Drug Alcohol Depend. 2015; 146: 30-8.

4. Lindholm P, Voutilainen MH, Lauren J, Peranen J, Leppanen VM, Andressoo $\mathrm{JO}$, et al. Novel neurotrophic factor CDNF protects and rescues midbrain dopamine neurons in vivo. Nature. 2007; 448: 73-7.

5. Hoffer BJ. Commentary on chronic infusion of CDNF prevents 6-OHDA-induced deficits in a rat model of Parkinson's disease. Merja H. Voutilainen et al. Experimental neurology. 2011; 230: 162-6.

6. Bjorklund A, Kirik D, Rosenblad C, Georgievska B, Lundberg C, Mandel RJ. Towards a neuroprotective gene therapy for Parkinson's disease: use of adenovirus, AAV and lentivirus vectors for gene transfer of GDNF to the nigrostriatal system in the rat Parkinson model. Brain Res. 2000; 886: 82-98.

7. Nagahara AH, Tuszynski MH. Potential therapeutic uses of BDNF in neurological and psychiatric disorders. Nature reviews Drug discovery. 2011; 10: 209-19.

8. Voutilainen MH, Back S, Peranen J, Lindholm P, Raasmaja A, Mannisto PT, et al. Chronic infusion of CDNF prevents 6-OHDA-induced deficits in a rat model of Parkinson's disease. Experimental neurology. 2011; 228: 99-108.

9. Ren X, Zhang T, Gong X, Hu G, Ding W, Wang X. AAV2-mediated striatum delivery of human CDNF prevents the deterioration of midbrain dopamine neurons in a 6-hydroxydopamine induced parkinsonian rat model. Experimental neurology. 2013; 248: 148-56.

10. Bowyer JF, Davies DL, Schmued L, Broening HW, Newport GD, Slikker W, Jr., et al. Further studies of the role of hyperthermia in methamphetamine neurotoxicity. The Journal of pharmacology and experimental therapeutics. 1994; 268: 1571-80.

11. Yamamoto BK, Moszczynska A, Gudelsky GA. Amphetamine toxicities: classical and emerging mechanisms. Annals of the New York Academy of Sciences. 2010; 1187: 101-21.

12. Wang L, Wang Z, Zhang F, Zhu R, Bi J, Wu J, et al. Enhancing Transgene Expression from Recombinant AAV8 Vectors in Different Tissues Using Woodchuck Hepatitis Virus Post-Transcriptional Regulatory Element. International journal of medical sciences. 2016; 13: 286-91.

13. Yu B, Wang C, Dong J, Zhang $\mathrm{M}$, Zhang $\mathrm{H}, \mathrm{Wu}$ J, et al. Chimeric hexon HVRs protein reflects partial function of adenovirus. Biochemical and biophysical research communications. 2012; 421: 170-6.

14. Zhong J, Fan S, Yan Z, Xiao S, Wan L, Chen C, et al. Effects of Nogo-A Silencing on TNF-alpha and IL-6 Secretion and TH Downregulation in Lipopolysaccharide-Stimulated PC12 Cells. BioMed research international. 2015; 2015: 817914.

15. Ares-Santos S, Granado N, Espadas I, Martinez-Murillo R, Moratalla R. Methamphetamine causes degeneration of dopamine cell bodies and terminals of the nigrostriatal pathway evidenced by silver staining. Neuropsychopharmacol. 2014; 39: 1066-80.

16. Sulzer D, Chen TK, Lau YY, Kristensen H, Rayport S, Ewing A. Amphetamine redistributes dopamine from synaptic vesicles to the cytosol and promotes reverse transport. J Neurosci. 1995; 15: 4102-8.

17. Broening $\mathrm{HW}, \mathrm{Pu} \mathrm{C}$, Vorhees CV. Methamphetamine selectively damages dopaminergic innervation to the nucleus accumbens core while sparing the shell. Synapse. 1997; 27: 153-60.

18. Cass WA. Decreases in evoked overflow of dopamine in rat striatum after neurotoxic doses of methamphetamine. The Journal of pharmacology and experimental therapeutics. 1997; 280: 105-13.

19. Haughey HM, Fleckenstein AE, Hanson GR. Differential regional effects of methamphetamine on the activities of tryptophan and tyrosine hydroxylase. J Neurochem. 1999; 72: 661-8.

20. Morgan ME, Gibb JW. Short-term and long-term effects of methamphetamine on biogenic amine metabolism in extra-striatal dopaminergic nuclei. Neuropharmacology. 1980; 19: 989-95

21. Harvey BK, Chou J, Shen H, Hoffer BJ, Wang Y. Diadenosine tetraphosphate reduces toxicity caused by high-dose methamphetamine administration. Neurotoxicology. 2009; 30: 436-44

22. Cass WA. GDNF selectively protects dopamine neurons over serotonin neurons against the neurotoxic effects of methamphetamine. J Neurosci. 1996; 16: 8132-9.

23. Cass WA, Peters LE, Harned ME, Seroogy KB. Protection by GDNF and other trophic factors against the dopamine-depleting effects of neurotoxic doses of methamphetamine. Ann Ny Acad Sci. 2006; 1074: 272-81.

24. Yang X, Liu Y, Liu C, Xie W, Huang E, Huang W, et al. Inhibition of ROCK2 expression protects against methamphetamine-induced neurotoxicity in PC12 cells. Brain Res. 2013; 1533: 16-25.
25. Klein RL, Dayton RD, Leidenheimer NI, Jansen K, Golde TE, Zweig RM. Efficient neuronal gene transfer with AAV8 leads to neurotoxic levels of tau or green fluorescent proteins. Molecular therapy : the journal of the American Society of Gene Therapy. 2006; 13: 517-27.

26. Cordero-Llana O, Houghton BC, Rinaldi F, Taylor H, Yanez-Munoz RJ, Uney $\mathrm{JB}$, et al. Enhanced Efficacy of the CDNF/MANF Family by Combined Intranigral Overexpression in the 6-OHDA Rat Model of Parkinson's Disease. Molecular Therapy. 2015; 23: 244-54

27. Cheng L, Zhao H, Zhang W, Liu B, Liu Y, Guo Y, et al. Overexpression of conserved dopamine neurotrophic factor (CDNF) in astrocytes alleviates endoplasmic reticulum stress-induced cell damage and inflammatory cytokine secretion. Biochemical and biophysical research communications. 2013; 435: 34-9.

28. Zhao H, Cheng L, Liu Y, Zhang W, Maharjan S, Cui Z, et al. Mechanisms of anti-inflammatory property of conserved dopamine neurotrophic factor: inhibition of JNK signaling in lipopolysaccharide-induced microglia. Journal of molecular neuroscience : MN. 2014; 52: 186-92.

29. Mei JM, Niu CS. Effects of CDNF on 6-OHDA-induced apoptosis in PC12 cells via modulation of $\mathrm{Bcl}-2 / \mathrm{Bax}$ and caspase-3 activation. Neurological sciences : official journal of the Italian Neurological Society and of the Italian Society of Clinical Neurophysiology. 2014; 35: 1275-80. 